This is the peer reviewed version of the following article:

Hortelão A.C., García-J imeno S., Cano-Sarabia M., Patiño T., Maspoch D., Sanchez S.. LipoBots: Using Liposomal Vesicles as Protective Shell of Urease-Based Nanomotors. Advanced Functional Materials, (2020). . : - .

10.1002/adfm.202002767,

which has been published in final form at https://dx.doi.org/10.1002/adfm.202002767. This article may be used for non-commercial purposes in accordance with Wiley Terms and Conditions for Use of Self-Archived Versions. 


\section{Lipobots: using liposomal vesicles as protective shell of urease-based nanomotors}

Ana C. Hortelão, ${ }^{\star}$ Sonia García-Jimeno, ${ }^{\dagger}$ Mary Cano-Sarabia, ${ }^{\dagger}$ Tania Patiño, ${ }^{\not}{ }^{*}{ }^{*}$ Daniel Maspoch ${ }^{\dagger *}$ and Samuel Sanchez ${ }^{\ddagger *}$

$¥$ A. C. Hortelão, Dr. T. Patiño, Prof. Dr. S. Sánchez

Institute for Bioengineering of Catalonia (IBEC), The Barcelona Institute of Science and Technology (BIST), Baldiri i Reixac 10-12, 08028 Barcelona Spain

'Dr. S. García-Jimeno, Dr. M. Cano-Sarabia, Prof. Dr. D. Maspoch

Catalan Institute of Nanoscience and Nanotechnology (ICN2), CSIC and The Barcelona Institute of Science and Technology, Universitat Autonoma de Barcelona, Bellaterra, 08193

Barcelona, Spain

"Dr. T. Patiño

Chemistry Department, University of Rome, Tor Vergata, Via della Ricerca Scientifica, 00133 Rome, Italy

*tpatino@ibecbarcelona.eu

§ Prof. Dr. D. Maspoch, Prod. Dr. S. Sánchez

Institució Catalana de Recerca i Estudis Avancats (ICREA), Passeig Lluís Companys 23, 08010 Barcelona, Spain

* ssanchez@ibecbarcelona.eu

* daniel.maspoch@icn2.cat

Keywords: nanomotors, enzymatic catalysis, self-propulsion, liposomes

Developing self-powered nanomotors made of biocompatible and functional components is of paramount importance in future biomedical applications. Herein we report the development of LipoBots (LBs) composed of liposomal carrier containing urease enzymes for propulsion.

Given the functional nature of liposomes, enzymes can be either encapsulated or coated on the surface of the vesicles. We first studied the influence of urease location on motion dynamics, finding that the surface-urease LBs underwent self-propulsion, whereas the encapsulatedurease LBs did not. However, adding a percolating agent present in the bile salts to the encapsulated-urease LBs triggered active motion is a larger magnitude. Moreover, we found that when we exposed both types of nanomotors to a medium of similar $\mathrm{pH}$ found in the stomach, the surface-urease LBs lost activity and motion capabilities, while the encapsulatedurease LBs gained activity and retained mobility. Our results on protection of an enzyme through encapsulation within liposomes, and in-situ triggering of the motion of LBs upon 
exposure to bile salts, may open new avenues for the use of liposome-based nanomotors in drug delivery, for example, in the gastrointestinal tract, where bile salts are naturally present in the intestine

\section{Introduction}

Over the past decade, scientists inspired by nature have developed nanoscale motors, known as catalytic nanomotors, that harvest chemical energy from their surrounding microenvironment and subsequently convert it into motion for various practical applications. Such devices have been reported to swim,,${ }^{[1-4]}$ transport, ${ }^{[5-8]}$ drill, ${ }^{[9-11]}$ clean, ${ }^{[12-18]}$ sense and actuate in fluids. ${ }^{[19-22]}$ Accordingly, they demonstrate great potential for biomedical applications. ${ }^{[23-28]}$ Indeed, compared to their passive counterparts, these actively propelled devices can cover larger areas/volumes, thus increasing potential interactions with a target; ${ }^{[29,30]}$ penetrate tissue to a greater degree; ${ }^{[29,31,32]}$ and perform superior drug delivery. ${ }^{[33,34]}$

An especially appealing aspect of using catalytic nanomotors for biomedical applications is the possibility of actuating them in situ using various biomolecules as fuels. In this regard, enzymes are an attractive source of catalytic power for nanomotors, as they are highly diverse, substrate-specific and ubiquitous in the body. Thus, entire libraries of enzyme/biomolecule (engine/fuel) combinations could be designed for specific on-demand applications. ${ }^{[35-37]}$ Enzymes used in catalytic nanomotors include urease, ${ }^{[38-44]}$ acetylcholine esterase, ${ }^{[44]}$ glucoseoxidase, ${ }^{[39,44,45]}$ lipase ${ }^{[46]}$ catalase ${ }^{[39,42,47-50]}$ and combinations thereof, all which can induce propulsion of various nano- and/or micro-particles. Nevertheless, the use of enzyme-powered nanomotors in vivo demands additional application requirements beyond biocompatibility and fuel bioavailability. For instance, inside living organisms, such devices could be exposed to harsh conditions (e.g. other enzymes, changes in $\mathrm{pH}$ ) that can degrade and consequently inactivate the enzymatic motor. ${ }^{[51]}$ 
An intriguing but scarcely explored strategy for improving the stability of enzymepowered nanomotors under harsh conditions is to protect the enzymes by encapsulating them into functional nanostructures: in other words, by using nanomaterials as both functional and protective chassis. In this study, we chose liposomes as preliminary chassis for three reasons: firstly, for their well-known capacity for encapsulation and protection of various actives, ranging from small drug molecules to larger biomolecules (e.g. proteins and enzymes); ${ }^{[52]}$ secondly, for their permeable membrane, which enables bidirectional transport of substances in and out of them; and finally, for their excellent biocompatibility and safety. ${ }^{[53]}$ To date, very little work has been done in this field. In a very recent and pioneering example, Sen and coworkers coupled enzymes onto the outer layer of liposomes, observing self-propulsion and chemotactic behavior in the resultant conjugates. ${ }^{[41,54]}$ The surface enzymes were sensitive to surrounding ionic gradients, and the direction of motion of the resultant nanomotors depended on the Hoffmeister series. The work by Sen and colleagues provided a foundation for using liposomes as chassis for enzyme-nanomotors; however, they did not explore encapsulation as an enzyme-protection strategy.

In our study, we demonstrate a new type of enzyme-powered nanomotors, called LipoBots (LBs), which are powered by urease. In these LBs, the urease is encapsulated into the inner liposomal compartment, named LB-I. For comparison purposes, we also synthesized a second prototype of LBs, LB-O, in which we electrostatically bound the urease to the outer lipid layers of the liposomes. We investigated how the location of the urease relative to the liposome influenced the motility and enzymatic activity of each type of LB under different conditions, such as harsh acidic environments as well as in the presence of sodium deoxycholate, a bile salt that is naturally present in the gastrointestinal tract. ${ }^{[55]}$ This salt acts as an edge activator, causing disruption and permeabilization of the lipid bilayer ${ }^{[56-58]}$ and therefore, enabling exchange of substrates and products. Accordingly, exposure of LB-I to sodium deoxycholate triggers motion of the nanomotor. As proof of concept, we exploited the 
protective effects of the lipid bilayer on the encapsulated enzymes, demonstrating that the enzymatic activity of these liposomal motors can be retained after incubation in harsh acidic conditions and their motion can be triggered by posterior addition of sodium deoxycholate at neutral $\mathrm{pH}$.

\section{Results and Discussion}

LipoBots were prepared using the thin-film hydration method. ${ }^{[59,60]}$ Briefly, 1,2-

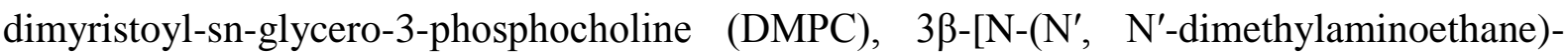
carbamoyl]cholesterol hydrochloride (Chol+) and cholesterol were dissolved in chloroform solutions and mixed at 1:0.5:0.5 molar ratio, respectively, at a total lipid concentration of 30 $\mathrm{mM}$. After, the organic solvent was removed under vacuum and nitrogen to afford a dry lipid film, which was hydrated under vigorous stirring with a $3 \mathrm{mg} / \mathrm{mL}$ urease solution for LB-I, or PBS for LB-O. Under these conditions, the stacks of liquid crystalline lipid bilayers become fluid and swell, resulting in their detachment during agitation and their self-closure to form multilamellar large vesicles (MLV). Small unilamellar vesicles were obtained by homogenizing the MLV suspension using an extruder and a polycarbonate membrane (pore size $200 \mathrm{~nm}$ ). After extrusion, the non-encapsulated urease from the LB-I sample was eliminated by centrifugation at $50000 \mathrm{rpm}$ and $4^{\circ} \mathrm{C}$ for 30 minutes and re-suspending the precipitated pellet with PBS. This step was performed three times. On the other hand, LB-O were prepared by incubating the extruded PBS-hydrated liposomes overnight with urease solution $(3 \mathrm{mg} / \mathrm{mL}$, in PBS) in the rotary shaker at room temperature. In this latter case, the unattached urease was also eliminated by centrifugation as described above (Figure 1a).

The morphology and size of both LBs were investigated using cryo-transmission electron microscopy (cryoTEM), which revealed the formation of a mixture of unilamellar and multivesicular vesicles with a mean radius of $105.7 \pm 0.8 \mathrm{~nm}$ and $100.7 \pm 0.5 \mathrm{~nm}$ (average \pm 
standard deviation, SD) for LB-O and LB-I, respectively (Figures 1b,c). The LBs were also characterized using Dynamic Light Scattering (DLS), where we observed that LB-O presented a broader peak than bare liposomes, that could be attributed to aggregation caused by the presence of enzymes on the surface of the liposomes.
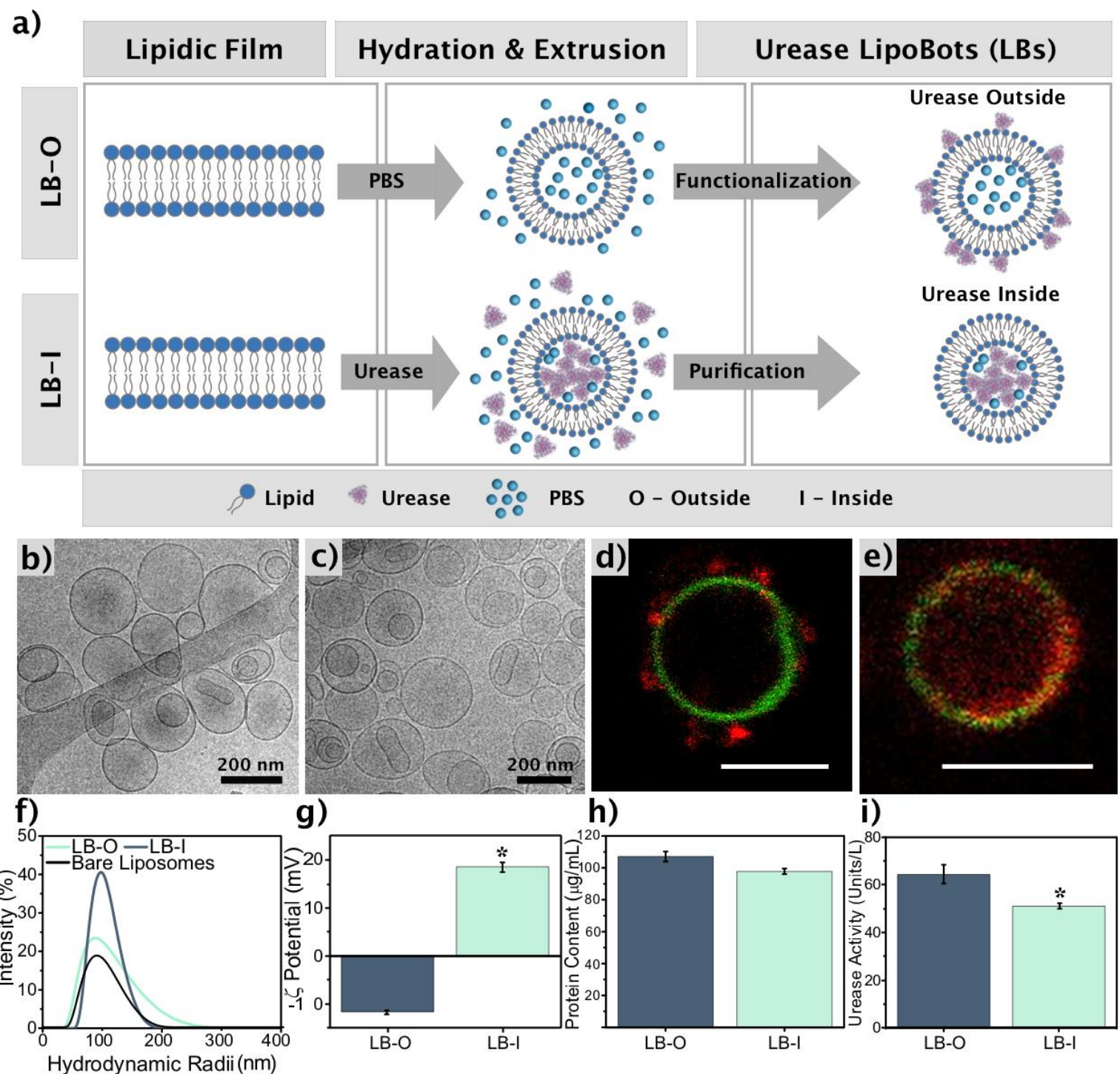

h)

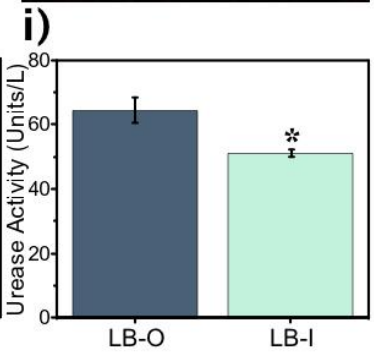

Figure 1. Fabrication and characterization of the LipoBots (LBs), comprising urease enzyme on the outer surface (LB-O), or into the inner compartment (LB-I). a) Scheme illustrating the fabrication process of LB-O and LB-I. Cryo-TEM images of b) LB-O and c) LB-I. Confocal laser scanning microscopy images of urease (red) in d) micron-sized LB-O (green) and e) micron-sized LB-I (green). Scale bars are $2 \mu \mathrm{m}$. f) Hydrodynamic radii characterization by DLS. g) Surface charge evaluation of the LB given by electrophoretic mobility measurements. h) 
Quantification of the total protein content present on the LBs. i) Enzymatic activity of the urease present on the LBs. Results are shown as the mean \pm standard error of the mean $(\mathrm{SEM}), \mathrm{N}=10$.

Enzyme localization and distribution was investigated using non-extruded micron-sized liposomes, due to the ease of visualization of the structures, and confocal laser scanning microscopy (CLSM), as displayed in Figures 1d and 1e. For this, we labeled urease enzymes with cyanine 5 and used Oregon Green 488 to mark the lipidic bilayer. We observed that, for LB-I, urease (in red) was mainly located into the liposomes (in green). In LB-O, the urease enzymes (in red) were located on the outer layer of the lipid membrane (in green). We also observed that the stochastic binding of the enzyme leads to the formation of asymmetric patches similar to those reported for silica-based micromotors using glutaraldehyde crosslinker. ${ }^{[43]} \mathrm{We}$ expect these observations to extrapolate to the smaller liposomes used in this work, however to further confirm the successful attachment of urease on the outer surface of liposomes in LB-O and encapsulation in LB-I, we investigated the surface charge of both LBs by studying their electrophoretic mobility (Figure 1g). LB-I showed to be positively charged $(18.6 \pm 1.0 \mathrm{mV}$, average \pm SEM) as it is composed of the cationic Chol+. ${ }^{[61]}$ Nevertheless, even though LB-O is also composed of Chol+, it displayed a negative surface charge $(-11.8 \pm 0.4 \mathrm{mV}$, average \pm SEM), which could be attributed to the urease negative charges at neutral $\mathrm{pH}$ (isoelectric point of urease ca. 5.1). ${ }^{[62]}$ Altogether, these results confirm that the urease molecules are mainly confined into the inner compartment in LB-I, and that urease molecules are attached on the external surface in LB-O.

We further characterized urease powered LBs by quantifying their total protein content (Figure 1h), using the bicinchoninic acid (BCA) assay, which relies on the reduction of copper by proteins and the colorimetric detection of the cuprous cation by BCA. ${ }^{[63]}$ The total amount protein was determined to be slightly higher in the case of LB-O (ca. 51\% binding yield) 
compared to LB-I (ca. 46\% encapsulation yield), nevertheless it was not statistically different $(\mathrm{P}<0.06$, unpaired t-test). Moreover, we studied how enzyme location affected its activity. Figure 1i shows urease activity of both types of LBs, evidencing that LB-I presents lower catalytic activity than LB-O (P < 0.05, unpaired t-test). This could be explained by not only the lower amount of urease molecules on the LB-I, but also to the fuel availability. Even though urea is a small water-soluble polar molecule, ${ }^{[64]}$ it relies on passive transport to cross lipid membranes, ${ }^{[65]}$ thus its access to urease in LB-O is much more facilitated than in the case of LB-I, leading to lower urease activity.

a)

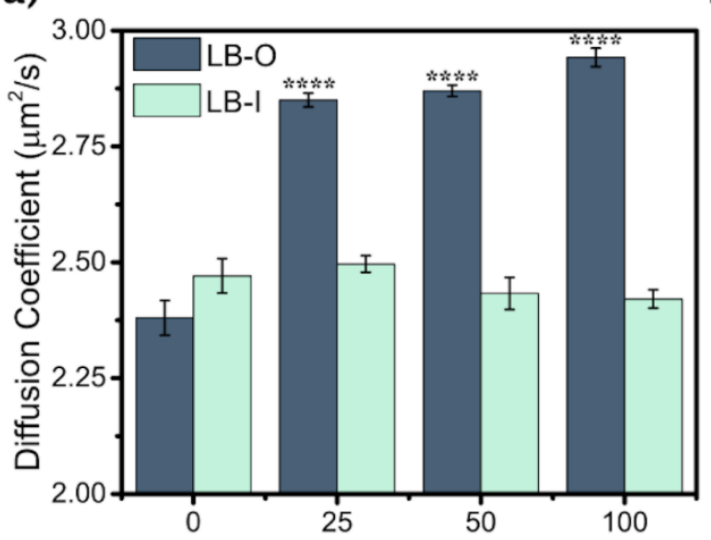

c)

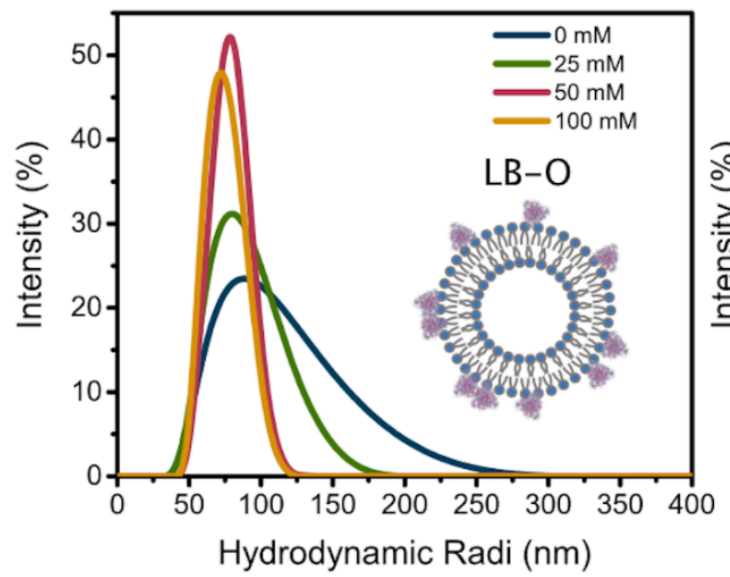

b)
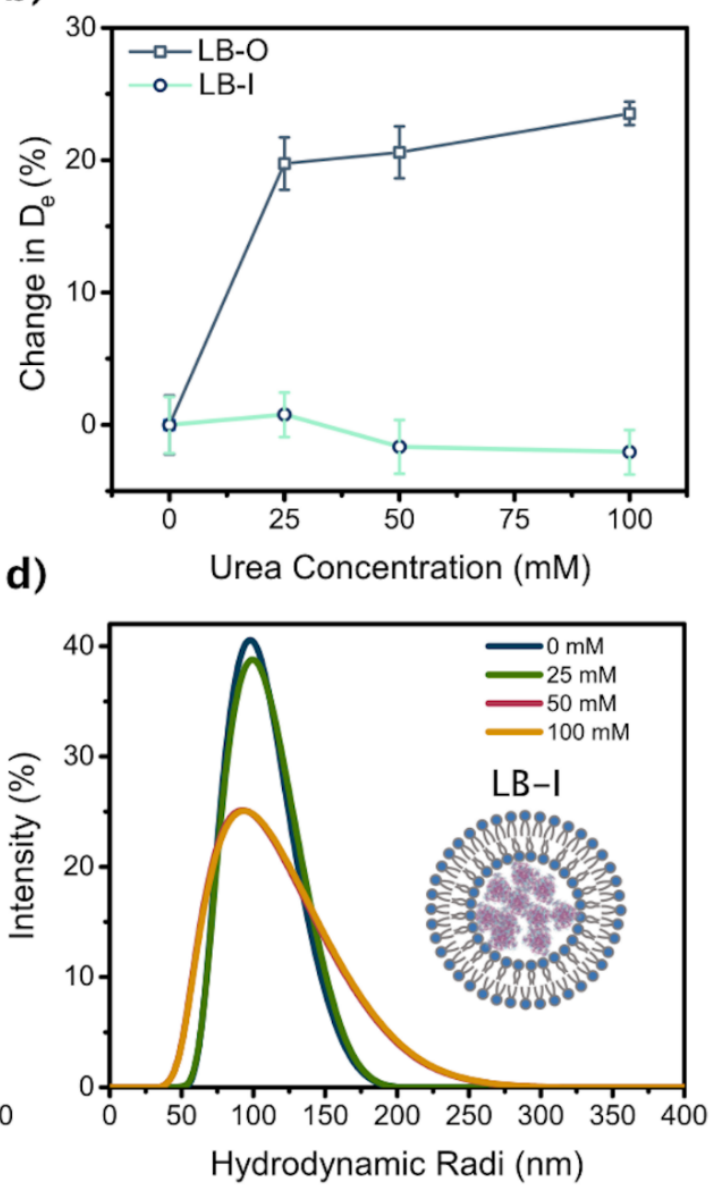

Figure 2. Motion profile of LB-O and LB-I. a) Diffusion coefficient of both LBs in the presence of increasing urea concentrations. Asterisks denote a significant difference from the control (0 $\mathrm{mM}$ ) with $P<0.0001$, results are shown as mean $\pm \mathrm{SEM}, \mathrm{N}=10 . \mathrm{b})$ Percentage of change in 
the diffusion coefficient in relation to the control $(0 \mathrm{mM}$ urea) for LB-O and LB-I. Hydrodynamic radii shift for c) LB-O and for d) LB-I.

We then investigated the motion capabilities of the urease-powered LBs by DLS, where the diffusion coefficient of the liposomes was obtained by fitting the correlation function. This function correlates the intensity of scattered light with the translational diffusion coefficient of the particles based on the premise that larger particles exhibit higher light scatter than smaller particles, due to Brownian dynamics. ${ }^{[66]}$ Figure 2a displays the diffusion coefficients of LB-O and LB-I when exposed to a range of urea concentrations $(25,50$ and $100 \mathrm{mM})$, evidencing an increase in the diffusion coefficient of LB-O with increasing urea concentrations. However, as seen in Figure 2b, the diffusion coefficient of LB-I did not increase in the presence of urea, but rather showed a slight decrease.

This could be attributed to the fact that, despite urea can be catalyzed by urease, the release of the reaction's products from the inner compartment of the liposomes is not efficient enough to generate motion or the release of products does not occur in an asymmetric manner. Furthermore, the slight decrease in diffusion coefficient could be explained by the possible accumulation of $\mathrm{CO} 2$ inside of the $\mathrm{LB}$, leading to its swelling, which in turn increases the hydrodynamic radii detected by DLS, traduced into a smaller diffusion. Figures $2 \mathrm{c}$ and $2 \mathrm{~d}$ show the shifts in hydrodynamic radii for LB-O and LB-I, respectively. For LB-O, a shift towards smaller hydrodynamic radii was observed with increasing concentrations of urea, evidencing also a narrower peak than the control without urea, suggesting a uniformity in the selfpropulsion. Oppositely, no shift was observed for the LB-I sample, indicating no selfpropulsion. Furthermore, when LB-I were exposed to $100 \mathrm{mM}$ of urea, the observed peak was broader than the control without urea, which could be explained by the retention of products within the inner cavity of the LBs, or variation in the release of catalytic products among the LB in the sample. 
As we previously observed, the absence of motion in LB-I could be explained because of the inefficient entrance of fuel urease into the liposomes and/or to the inefficient release of substances $\left(\mathrm{CO}_{2}\right.$ and $\left.\mathrm{NH}_{3}\right)$ resulting from the enzymatic reaction out of the liposomes. For this reason, we hypothesized that enhancement of the permeability of LB-I should facilitate the bidirectional transport of urea into the liposome and of $\mathrm{CO}_{2}$ and $\mathrm{NH}_{3}$ out of the liposome, opening a way for triggering their motion. In this respect, a well-known strategy to enhance the permeability of liposomes is by the use of bile salts. For instance, the bile salt sodium deoxycholate can act as an edge activator and disruptor of lipid bilayers by solubilization, causing an increase on bilayer flexibility and permeability. ${ }^{[67,68]}$ To study the effect of sodium deoxycholate on the permeability and self-propulsion capabilities of LB-I, we exposed them to different concentrations of sodium deoxycholate and investigated their motion capability by DLS.

a)

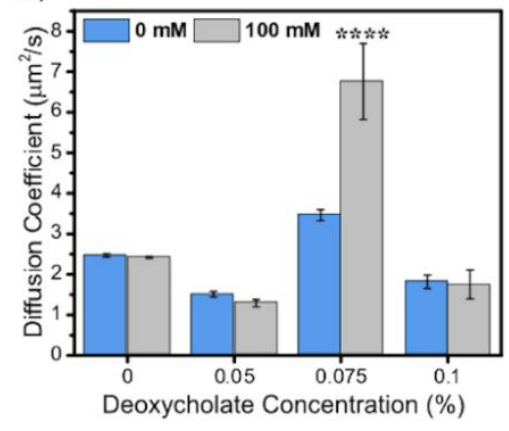

LB-I + Urea

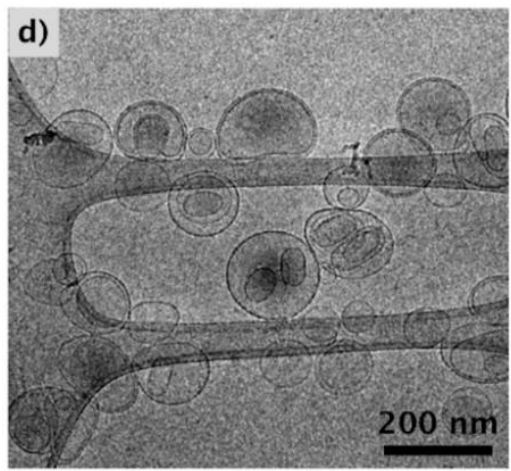

b)

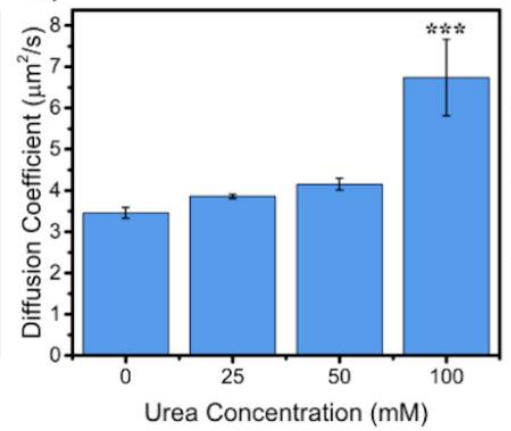

LB-I + Deoxycholate + Urea

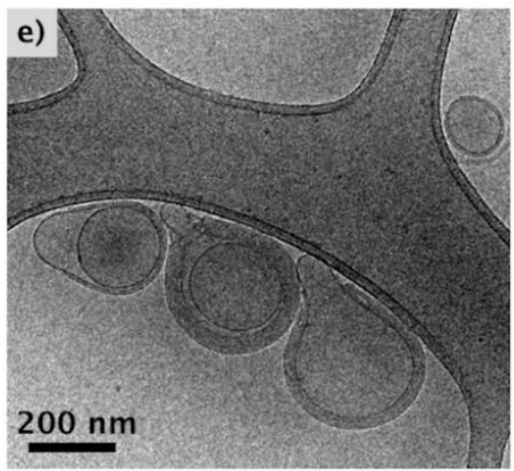

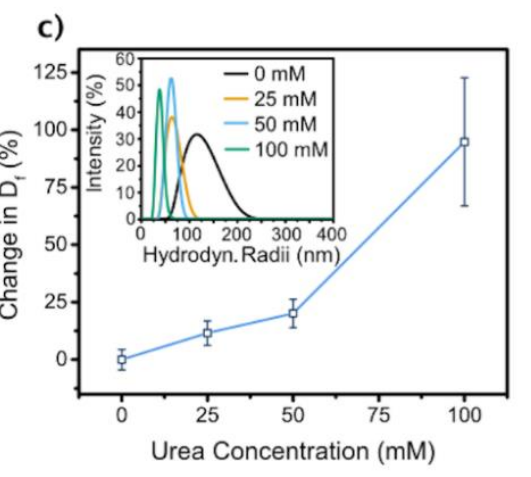

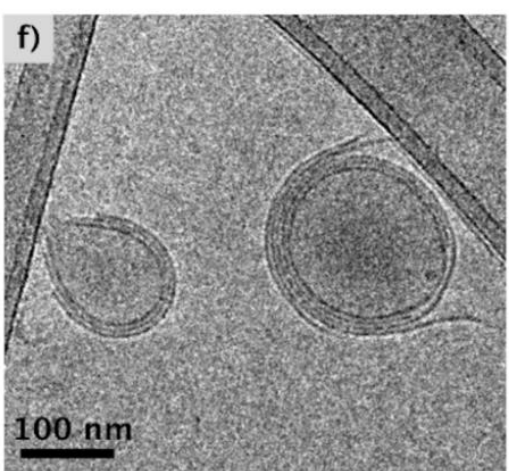

Figure 3. Motion profile of LB-I in the presence of sodium deoxycholate. a) Diffusion coefficient of the LB-I in the presence of different sodium deoxycholate concentrations. Asterisks denote a significant difference from control $(0 \mathrm{mM})$ with $P<0.001$, results are shown 
as mean $\pm \mathrm{SEM}, \mathrm{N}=10 . \mathrm{b}$ ) Diffusion coefficient of LB-I in the presence of $0.075 \%$ sodium deoxycholate and increasing concentrations of urea. Asterisks denote a significant difference from control $(0 \mathrm{mM})$ with $P<0.01$, results are shown as mean $\pm \mathrm{SEM}, \mathrm{N}=10$. c) Percentage of change in the diffusion coefficient of LB-I in relation to the control $(0.075 \%$ sodium deoxycholate, $0 \mathrm{mM}$ urea), inset: hydrodynamic radii of LB-I in the presence of $0.075 \%$ sodium deoxycholate and increasing concentrations of urea. CryoTEM images of d) LB-I and e, f) LBI in the presence of both deoxycholate and urea $(100 \mathrm{mM})$.

Figure 3a and Figure S1 show the DLS study of the diffusion coefficient of LB-I in the presence of the different sodium deoxycholate concentrations, both in the absence and presence of urea $(100 \mathrm{mM})$ We found that, at a sodium deoxycholate concentration of $0.05 \%$, the LB-I still show low diffusion coefficients, meaning that urea does not induce self-propulsion. This fact could be explained because LB-I are not enriched enough with deoxycholate molecules to increase the lipid bilayer permeability. Nonetheless, upon increasing the concentration of sodium deoxycholate up to $0.075 \%$, a higher diffusion coefficient was clearly observed, being it significantly higher when exposed to urea. At higher concentrations of sodium deoxycholate $(0.1 \%)$, however, we observed a decrease of the diffusion coefficient, both in the absence and presence of urea. We attributed this to the fact that, when the cholate concentration increases, the increase in fluidity and deformability of the bilayers leads to fusion among liposomes, as well as formation of smaller mixed micelles. ${ }^{[58,69]}$

Being confirmed the possibility to in-situ trigger the active motion of urease-encapsulated LB-I upon exposure to sodium deoxycholate and taking the concentration of $0.075 \%$ as the optimal concentration of this bile salt, we then investigated the self-propulsion of LB-I with respect to fuel concentration (Figures 3b-c and Figure S2). To this end, LB-I were incubated with increasing concentrations of urea, ranging from $0 \mathrm{mM}$ to $100 \mathrm{mM}$, where a concentrationdependent increase of the diffusion coefficient was observed. Since sodium deoxycholate alters 
the fluidity and permeability of the lipid bilayer, we studied the integrity of LB-I upon exposure to sodium deoxycholate by cryoTEM (Figures 3d-f). Figures $3 \mathrm{e}$ and f revealed the presence of liposomes with a mean radius of $107.7 \pm 3.6 \mathrm{~nm}$ (average \pm SEM), thus confirming the stability of LB-I under motion-triggering conditions. Moreover, a comparison of LB-I incubated in PBS containing urea in the presence and absence of sodium deoxycholate showed that liposomes were more elongated in presence of sodium deoxycholate, further suggesting a higher flexibility (and thus, permeability) of LB-I under these conditions, as reported in literature. ${ }^{[56]}$

a)
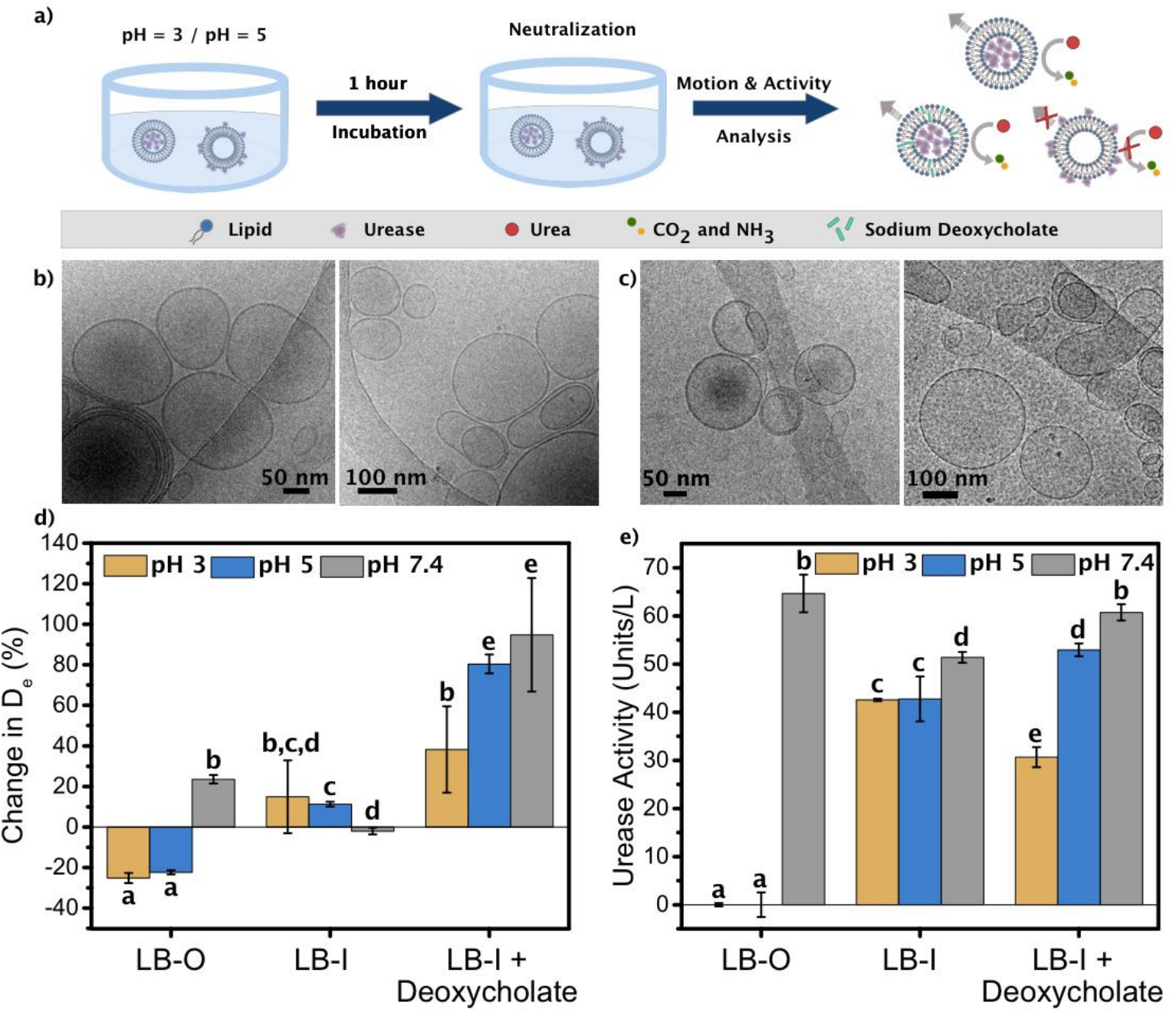

Figure 4. Stability in acidic conditions and recovery of the motion capabilities of LBs. a) Scheme illustrating the experimental approach: LBs incubation in acidic conditions for 1 hour, followed by neutralization and investigation of their motion abilities. CryoTEM images of b) LB-O and c) LB-I after incubation in acidic conditions. d) Percentage change in the diffusion 
coefficient in relation to control, for LB-O, LB-I and LB-I in the presence of $0.075 \%$ of sodium deoxycholate, results are shown as mean \pm SEM, $N=5$ e) Enzymatic activity of urease present in the LBs after neutralization, results are shown as mean $\pm \mathrm{SEM}, \mathrm{N}=3$. Different superscripts denote groups of significance $(P<0.05)$

The possibility to fabricate enzyme-encapsulated nanomotors which motion is activated under certain conditions opens new avenues for using this class of nanomotors in conditions in which the enzymes can degrade. This enzyme-protection strategy uses first the liposomal chassis to protect the enzymes when crossing adverse conditions, making them still active when the motion triggering conditions are found or applied. As proof-of-concept, we investigated this concept by exposing LB-I (and also LB-O for comparison purposes) to an acidic medium of the same $\mathrm{pH}$ as stomach for 1 hour (Figures $4 \mathrm{a}-\mathrm{c}$ ). The acidity of the stomach is variable throughout the day and dependent on the food intake, with $\mathrm{pH}$ values ranging from 2 to $6 ;^{[55]}$ therefore $\mathrm{pH}$ 3 and $\mathrm{pH} 5$ were chosen to mimic different acidity levels of the stomach. ${ }^{[55,70]}$

After 1-hour incubation at $\mathrm{pH} 3$ and 5, both LB-I and LB-O solutions were neutralized (final $\mathrm{pH}=7.2)$ by adding PBS, PBS containing urea $(100 \mathrm{mM})$, PBS containing $0.075 \%$ sodium deoxycholate, or PBS containing both urea and sodium deoxycholate (see methods in SI for further details) and analyzed by DLS to investigate their ability to retain motility and enzymatic activity after incubation in acidic conditions. Figures $4 \mathrm{~d}$ and e display the change in diffusion coefficient in the presence of urea $(100 \mathrm{mM})$, in relation to control (0 $\mathrm{mM}$ urea), and the enzymatic activity of both types of LBs when exposed to $\mathrm{pH} 3$ and 5. For comparison purposes, they also show the change in diffusion coefficient and enzymatic activity of both LBs directly exposed to $\mathrm{pH}=7.4$. We observed that, unlike at $\mathrm{pH}=7.4$, the enzymatic activity and ability to self-propel of LB-O are completely lost after incubation at both $\mathrm{pH} 3$ and 5, which can be due to the denaturalization of the urease placed on the external surface of the liposomes, or even to the detachment of the enzyme from the liposome's surface since it is bound by electrostatic interactions and the acidic environment causes its surface charge to turn positive. 
The enzymatic activity of LB-O was also measured, from which a complete loss of activity was detected. Remarkably, LB-I displayed a slight increase in diffusion coefficient in the presence of urea after incubation in acidic conditions, even without addition of sodium deoxycholate. This can be due to an effect of acidic conditions in the permeability of the lipid bilayer, allowing for a more efficient exchange of substrate and catalytic products. Nevertheless, in the presence of both urea and sodium deoxycholate, LB-I presented a higher increase in diffusion coefficient, indicating retention of their self-propulsion abilities and the protection of urease from denaturalization inside the liposomes after incubation in acidic conditions. This protection was also confirmed by measuring the enzymatic activity of LB-I after incubation in acidic conditions, observing that urease enzymes remained active (Figure 4e).

\section{Conclusion}

In summary, we have developed two different liposome-based nanomotors powered by urease, which were either encapsulated or electrostatically bound to the outer surface of the liposome. We verified that the stochastic electrostatic binding of urease on the outer surface of the liposomes is sufficient to generate an asymmetric distribution that can lead to motion. Upon the presence of the sodium deoxycholate to act as an edge activator, the fluidity of the lipid bilayer is modulated, and this enhances the motility of the LBs containing encapsulated urease. Moreover, we demonstrated the protective effect of the lipid bilayer shell surrounding the enzymes, showing that after 1 hour incubation in harsh conditions such as acidic $\mathrm{pH}$, the LipoBots are able to retain enzymatic activity and motility.

Upon the presence of the sodium deoxycholate to act as an edge activator, the fluidity of the lipid bilayer is modulated, and this enhances the motility of the LBs containing encapsulated urease. Moreover, the lipid bilayer surrounding the encapsulated enzymes exerts a protective effect against harsh conditions such as acidic $\mathrm{pH}$, allowing the liposomal nanomotors to recover motility after a 1-hour incubation period in these conditions. Further studies focusing on the in 
vivo kinetics of these liposomal based nanomotors and in the presence of gradients of enzymatic substrate and sodium deoxycholate are required. Nevertheless, these findings can pave the way to the development of new drug delivery systems comprising enzyme-powered nanomotors.

\section{Experimental Section}

\section{Materials}

1,2-dimyristoyl-sn-glycero-3-phosphocholine (DMPC) was purchased from Lipoid GmbH@ (Switzerland). $\quad 3 \beta-\left[\mathrm{N}-\left(\mathrm{N}^{\prime}, \mathrm{N}^{\prime}\right.\right.$-dimethylaminoethane)-carbamoyl]cholesterol hydrochloride (Chol+) cholesterol $(\mathrm{CH})$, urease (from Canavalia ensiformis, type IX, powder, 50000 - 100 000 units per gram of solid), Urease Activity Assay Kit, urea, and sodium deoxycholate were acquired from Sigma Aldrich (USA). Oregon Green 488 DHPE dye, BCA Protein Assay Kit, and phosphate-buffered saline (PBS) were supplied by Thermo Fisher Scientific (USA). Spectra/Por 7 Standard RC pretreated Dialysis Tubing (3.5 kDa) was purchased from Spectrum. Cyanine5 NHS ester (Cy5) was purchased from Lumiprobe.

\section{Instruments}

The liposomes were prepared using an extruder from Lipex Biomembranes, Canada. Protein quantification and enzymatic activity assays were carried out using an Infinite M200 PRO Multimode Microplate Reader. Cryo-transmission electron microscope (CryoTEM) images were obtained using a JEOL-JEM 1400 microscope (Jeol Ltd., Tokyo, Japan). Confocal laser scanning microscope (CLSM) images were obtained using a Leica TCS SP5 confocal laser scanning microscope (Leica Microsystems, Germany). Hydrodynamic radii and electrophoretic mobility measurements were performed using a Wyatt Möbius and a Malvern Zetasizer.

\section{Preparation of the $L B S$}


Liposomal-based nanomotors (LBs) were prepared using the thin-film hydration method. Two LB prototypes with different enzyme position, inside (LB-I) and outside (LB-O) were developed. Briefly, DMPC, Chol+ and Chol were dissolved in chloroform solutions and mixed at 1:0.5:0.5 molar ratio, respectively. Total lipid concentration was $30 \mathrm{mM}$. The organic solvent was removed under vacuum and nitrogen to afford a dry lipid film, which was hydrated under vigorous stirring with a solution of urease dissolved in PBS (3 mg/mL) for LB-I, or PBS for LB-O. Under these conditions, the stacks of liquid crystalline lipid bilayers become fluid and swell, which led to detachment during agitation and selfclosure to yield multilamellar large vesicles (MLV). After, the MLV were homogenized using an extruder and a polycarbonate membrane (pore size $200 \mathrm{~nm}$ ). For LB-I, the non-encapsulated urease was eliminated washing by centrifugation at $50000 \mathrm{rpm}$ and $10^{\circ} \mathrm{C}$ for $30 \mathrm{~min}$ and re-suspending the precipitated pellet with PBS thrice. For LB-O, the PBS-hydrated liposomes were incubated overnight with a $3 \mathrm{mg} / \mathrm{mL}$ urease PBS solution in the rotary shaker at $25^{\circ} \mathrm{C}$. Finally, the unbound urease was eliminated washing by centrifugation as described above.

The micron-sized LBs were prepared using the same protocol described above, omitting the extrusion step.

Characterization of size distribution, surface charge and morphology of LBs

Particle size distributions of the liposomes were determined using a dynamic light scattering (DLS) analyzer combined with noninvasive backscatter technology. The stability of the liposomes was examined by measuring their electrophoretic mobility using a Malvern Zetasizer. For this, the liposome-based nanomotors were diluted 50 times and placed in a cuvette for analysis. Liposome morphology was examined using Cryo-Transmission Electron Microscopy (Cryo-TEM) in a JEOL-JEM 1400 microscope (Jeol Ltd., Tokyo, Japan). For this, LB-I in each condition before being placed in the TEM grid and cryogenized for observation.

\section{Functionalization of urease with cy5}

A solution of urease $(20 \mathrm{mg} / \mathrm{mL})$ in sodium bicarbonate buffer $(100 \mathrm{mM})$. Next, $7 \mu \mathrm{L}$ of a Cy 5 solution in DMSO $(5 \mathrm{mM})$ were added, and the mixture was incubated for $4 \mathrm{~h}$ at $25{ }^{\circ} \mathrm{C}$ and 
shaking in the dark. The solution of labeled urease was then dialyzed in PBS (3.5 kDa pore membrane) for $24 \mathrm{~h}$ to eliminate nonreacted Cy5 molecules.

\section{Evaluation of urease distribution on the LBS}

Evaluation of the distribution of urease in liposomes was done by CLSM. For that, fluorescently labelled urease was encapsulated in liposomes for LB-I or incubated with liposomes for LB-O, with the lipid bilayer previously labelled with Oregon Green 488 DHPE. The resulting non-extruded liposomal formulations were examined by CLSM.

\section{Total protein content quantification assays}

The concentration of urease present on the LBs was measured using the BCA Protein Assay Kit from Thermo Fisher Scientific according to the manufacturer's instructions. This kit correlates the quantity of proteins with the reduction of copper by peptide bonds. ${ }^{[63]}$ For this, the total protein content present on the supernatants from liposome washings, as well as in the urease stock solutions used for incubation was measured. The total protein content bound or encapsulated in the liposomes was calculated as the difference between the content of the stock solutions and the content of the supernatants.

\section{Urease enzymatic activity assays}

Enzymatic activity of urease present on the LBs was evaluated using a commercial kit that determines the concentration of ammonia generated by Berthelot's method. ${ }^{[71]}$ The liposomal nanomotors were diluted 50 times, and the experiment was performed according to the manufacturer's instructions.

\section{Motion Behavior Analysis}

The motion behavior of the LipoBots was analyzed by DLS, a technique that correlates the intensity of scattered light with the translational diffusion coefficient of the particles. 
The purified LB-O were diluted 100 times prior to analysis by DLS, yielding dispersions containing liposomes and urea, at concentrations of $0 \mathrm{mM}, 25 \mathrm{mM}, 50 \mathrm{mM}$ and $100 \mathrm{mM}$. Each condition was measured at least 10 times. Regarding the LB-I, firstly they were analyzed in the absence of sodium deoxycholate using the methodology described above. For the analysis in the presence of sodium deoxycholate, this edge activator was added to the dispersions containing LB-I and urea before analysis, at concentrations of $0 \%, 0.05 \%, 0.075 \%$ and $0.1 \%(\mathrm{w} / \mathrm{w})$. Each condition was measured at least 10 times. The results were analyzed statistically by performing an ANOVA followed by Tukey's test.

\section{Motion Behavior after GI tract simulation}

The LBs were diluted 50 times in acidic solutions of $\mathrm{pH} 3$ and $\mathrm{pH} 5$ and incubated for $1 \mathrm{~h}$, in order to simulate the harsh stomach conditions. Following this, the liposomal dispersions were neutralized using PBS or PBS containing urea $(100 \mathrm{mM})$ for LNM-O, and with PBS, PBS containing sodium deoxycholate $(0.075 \% \mathrm{w} / \mathrm{w})$, or PBS containing both urea $(100 \mathrm{mM})$ and sodium deoxycholate $(0.075 \% \mathrm{w} / \mathrm{w})$ for LB-I. The neutralized dispersions were analyzed by DLS to evaluate their motion profiles. The results were analyzed statistically by performing an ANOVA followed by Tukey’s test.

\section{Supporting Information}

Supporting Information is available from the Wiley Online Library or from the author.

\section{Acknowledgements}

M. C.-S. and S. G-J. synthesized the liposome vesicles and encapsulated the enzymes. S. G.-J. performed the CSLM experiments. A. C. H and S. G.-J. performed the TEM characterization and the motility experiments. A. C. H. performed the protein quantification and enzymatic activity experiments and analyzed the data. D. M., S. S. and T. P. designed and supervised the work. The manuscript was written through contributions of all authors. All authors have given approval to the final version of the manuscript.

The research leading to these results has received funding from the Spanish MINECO (BOTSinFluids project), the Foundation BBVA (MEDIROBOTS project), the CERCA program by the Generalitat de Catalunya and the CaixaImpulse program by La Caixa Foundation (TERANOBOTS project). A.C.H. thanks MINECO for the Severo Ochoa PhD fellowship, and T.P. thanks the European Union's Horizon 2020 research and innovation program, under the Marie Skłodowska-Curie Individual Fellowship (H2020-MSCA-IF2018, DNA-bots). This work was also supported by the Catalan AGAUR (project 2017 SGR 238). It was also funded by the CERCA Program/Generalitat de Catalunya. ICN2 is supported by the Severo Ochoa program from the Spanish MINECO (Grant No. SEV-2017-0706).

((Acknowledgements, general annotations, funding. Other references to the title/authors can also appear here, such as "Author 1 and Author 2 contributed equally to this work.")) 
Received: ((will be filled in by the editorial staff))

Revised: ((will be filled in by the editorial staff)) Published online: ((will be filled in by the editorial staff))

References

[1] A. C. Balazs, P. Fischer, A. Sen, Acc. Chem. Res. 2018, 51, 2979.

[2] H. Wang, M. Pumera, Chem. Rev. 2015, 115, 8704.

[3] J. Katuri, X. Ma, M. M. Stanton, S. Sanchez, Acc. Chem. Res. 2016, 50, 2.

[4] Y. Ji, X. Lin, Z. Wu, Y. Wu, W. Gao, Q. He, Angew. Chemie Int. Ed. 2019, 58, 12200.

[5] T. Kroupa, S. Hermanová, C. C. Mayorga-Martinez, F. Novotný, Z. Sofer, M. Pumera, Langmuir 2019, 35, 10618.

[6] Y. Yoshizumi, K. Okubo, M. Yokokawa, H. Suzuki, Langmuir 2016, 32, 9381.

[7] J. Orozco, B. Jurado-Sánchez, G. Wagner, W. Gao, R. Vazquez-Duhalt, S. Sattayasamitsathit, M. Galarnyk, A. Cortés, D. Saintillan, J. Wang, Langmuir 2014, 30, 5082 .

[8] L. Baraban, M. Tasinkevych, M. N. Popescu, S. Sanchez, S. Dietrich, O. G. Schmidt, Soft Matter 2012, 8, 48.

[9] W. Xi, A. A. Solovev, A. N. Ananth, D. H. Gracias, S. Sanchez, O. G. Schmidt, Nanoscale 2013, 5, 1294.

[10] A. A. Solovev, W. Xi, D. H. Gracias, S. M. Harazim, C. Deneke, S. Sanchez, O. G. Schmidt, ACS Nano 2012, 6, 1751.

[11] S. K. Srivastava, M. Medina-Sánchez, B. Koch, O. G. Schmidt, Adv. Mater. 2016, 28, 832.

[12] Y. Ying, M. Pumera, Chem. - A Eur. J. 2019, 25, 106.

[13] S. Wang, Z. Jiang, S. Ouyang, Z. Dai, T. Wang, ACS Appl. Mater. Interfaces 2017, 9, 23974.

[14] M. Zarei, M. Zarei, Small 2018, 14, 1.

[15] M. Safdar, J. Simmchen, J. Jänis, Environ. Sci. Nano 2017, 4, 1602.

[16] J. Parmar, D. Vilela, K. Villa, J. Wang, S. Sánchez, J. Am. Chem. Soc. 2018, 140, 9317.

[17] M. Safdar, S. U. Khan, J. Jänis, Adv. Mater. 2018, 30, 1703660.

[18] F. Mushtaq, X. Chen, S. Staufert, H. Torlakcik, X. Wang, M. Hoop, A. Gerber, X. Li, J. Cai, B. J. Nelson, S. Pané, J. Mater. Chem. A 2019, 7, 24847.

[19] T. Patino, A. Porchetta, A. Jannasch, A. Lladó, T. Stumpp, E. Schäffer, F. Ricci, S. Sánchez, Nano Lett. 2019, 19, 3440.

[20] J. Orozco, V. García-Gradilla, M. D’Agostino, W. Gao, A. Cortés, J. Wang, ACS Nano 2013, 7, 818.

[21] V. V Singh, K. Kaufmann, J. Orozco, J. Li, M. Galarnyk, G. Arya, J. Wang, Chem. Commun. 2015, 51, 11190.

[22] L. Kong, J. Guan, M. Pumera, Curr. Opin. Electrochem. 2018, 10, 174.

[23] J. Li, B. Esteban-Fernández de Ávila, W. Gao, L. Zhang, J. Wang, Sci. Robot. 2017, 2.

[24] F. Peng, Y. Tu, D. A. Wilson, Chem. Soc. Rev. 2017, 46, 5289.

[25] C. Gao, Z. Lin, X. Lin, Q. He, Adv. Ther. 2018, 1, 1800056.

[26] Z. Lin, X. Fan, M. Sun, C. Gao, Q. He, H. Xie, ACS Nano 2018, 12, 2539.

[27] B. Esteban-Fernández de Ávila, P. Angsantikul, J. Li, W. Gao, L. Zhang, J. Wang, Adv. Funct. Mater. 2018, 28, 1705640.

[28] Z. Wu, L. Li, Y. Yang, P. Hu, Y. Li, S.-Y. Yang, L. V Wang, W. Gao, Sci. Robot. 2019, 4, eaax0613.

[29] A. C. Hortelao, R. Carrascosa, N. Murillo-Cremaes, T. Patino, S. Sánchez, ACS Nano 2019, 13, 429. 
[30] X. Wang, X.-Z. Chen, C. C. J. Alcântara, S. Sevim, M. Hoop, A. Terzopoulou, C. de Marco, C. Hu, A. J. de Mello, P. Falcaro, S. Furukawa, B. J. Nelson, J. Puigmartí-Luis, S. Pané, Adv. Mater. 2019, 31, 1970192.

[31] F. Peng, Y. Men, Y. Tu, Y. Chen, D. A. Wilson, Adv. Funct. Mater. 2018, 28, 1706117.

[32] J. Min, Y. Yang, Z. Wu, W. Gao, Adv. Ther. 2019, n/a, 1900125.

[33] A. C. Hortelão, T. Patiño, A. Perez-Jiménez, À. Blanco, S. Sánchez, Adv. Funct. Mater. 2018, 28, 1705086.

[34] A. Llopis-Lorente, A. Garciá-Fernández, N. Murillo-Cremaes, A. C. Hortelaõ, T. Patinõ, R. Villalonga, F. Sancenón, R. Martínez-Máñez, S. Sánchez, ACS Nano 2019, $13,12171$.

[35] X. Ma, A. C. Hortelão, T. Patiño, S. Sánchez, ACS Nano 2016, 10, 9111.

[36] T. Patiño, X. Arqué, R. Mestre, L. Palacios, S. Sánchez, Acc. Chem. Res. 2018, 51, 2662.

[37] M. Nijemeisland, L. K. E. A. Abdelmohsen, W. T. S. Huck, D. A. Wilson, J. C. M. van Hest, ACS Cent. Sci. 2016, $2,843$.

[38] X. Ma, X. Wang, K. Hahn, S. Sánchez, ACS Nano 2016, 10, 3597.

[39] X. Ma, A. Jannasch, U.-R. Albrecht, K. Hahn, A. Miguel-López, E. Schäffer, S. Sánchez, Nano Lett. 2015, 15, 7043.

[40] X. Ma, A. C. Hortelao, A. Miguel-López, S. Sánchez, J. Am. Chem. Soc. 2016, 138, 13782.

[41] S. Ghosh, F. Mohajerani, S. Son, D. Velegol, P. J. Butler, A. Sen, Nano Lett. 2019, 19, 6019.

[42] A. Somasundar, S. Ghosh, F. Mohajerani, L. N. Massenburg, T. Yang, P. S. Cremer, D. Velegol, A. Sen, Nat. Nanotechnol. 2019, 14.

[43] T. Patiño, N. Feiner-Gracia, X. Arqué, A. Miguel-López, A. Jannasch, T. Stumpp, E. Schäffer, L. Albertazzi, S. Sánchez, J. Am. Chem. Soc. 2018, 140, 7896.

[44] X. Arqué, A. Romero-Rivera, F. Feixas, T. Patiño, S. Osuna, S. Sánchez, Nat. Commun. 2019, 10, 1.

[45] L. K. E. A. Abdelmohsen, M. Nijemeisland, G. M. Pawar, G. A. Janssen, R. J. M. Nolte, J. C. M. van Hest, D. A. Wilson, ACS Nano 2016, 10, 2652.

[46] L. Wang, A. C. Hortelão, X. Huang, S. Sánchez, Angew. Chemie Int. Ed. 2019, 58, 7992.

[47] J. Sun, M. Mathesh, W. Li, D. A. Wilson, ACS Nano 2019, 13, 10191.

[48] B. J. Toebes, F. Cao, D. A. Wilson, Nat. Commun. 2019101 2019, 10, 1.

[49] B. J. Toebes, L. K. E. A. Abdelmohsen, D. A. Wilson, Polym. Chem. 2018, 9, 3190.

[50] Y. Wu, X. Lin, Z. Wu, H. Möhwald, Q. He, ACS Appl. Mater. Interfaces 2014, 6, 10476.

[51] S. K. Srivastava, G. Clergeaud, T. L. Andresen, A. Boisen, Adv. Drug Deliv. Rev. 2019, 138, 41.

[52] M. T. Hussain, N. Forbes, Y. Perrie, K. P. Malik, C. Duru, P. Matejtschuk, Int. J. Pharm. 2020, 573, 118722.

[53] Y. S. Youn, Y. H. Bae, Adv. Drug Deliv. Rev. 2018, 130, 3.

[54] A. Somasundar, S. Ghosh, F. Mohajerani, L. N. Massenburg, T. Yang, P. S. Cremer, D. Velegol, A. Sen, Nat. Nanotechnol. 2019, 14.

[55] D. M. Mudie, G. L. Amidon, G. E. Amidon, Mol. Pharm. 2010, 7, 1388.

[56] A. De La Maza, A. M. Manich, J. L. Parra, J. Microsc. 1997, 186, 75.

[57] H. Ahyayauch, M. Bennouna, A. Alonso, F. M. Goñi, Langmuir 2010, 26, 7307.

[58] L. G. Hermida, M. Sabés-Xamaní, R. Barnadas-Rodríguez, Soft Matter 2014, 10, 6677.

[59] A. D. Bangham, M. W. Hill, N. G. A. Miller, Korn, E. D., Ed.; Springer US: Boston, MA, 1974; pp. 1-68. 
[60] F. Szoka, D. Papahadjopoulos, Annu. Rev. Biophys. Bioeng. 1980, 9, 467.

[61] Y. Maitani, S. Igarashi, M. Sato, Y. Hattori, Int. J. Pharm. 2007, 342, 33.

[62] J. B. Sumner, D. B. Hand, J. Am. Chem. Soc. 1929, 149, 1925.

[63] P. K. Smith, R. I. Krohn, G. T. Hermanson, A. K. Mallia, F. H. Gartner, M. D. Provenzano, E. K. Fujimoto, N. M. Goeke, B. J. Olson, D. C. Klenk, Anal. Biochem. 1985, 150, 76.

[64] L. A. Pinck, M. A. Kelly, J. Am. Chem. Soc. 1925, 47, 2170.

[65] J. D. Harvey Lodish, Arnold Berk, S Lawrence Zipursky, Paul Matsudaira, David Baltimore, In Molecular Cell Biology; 2000; p. Chapter 15.

[66] J. Stetefeld, S. A. McKenna, T. R. Patel, Biophys. Rev. 2016, 8, 409.

[67] E. P. O. Silva, L. P. Franchi, A. C. Tedesco, RSC Adv. 2016, 6, 79631.

[68] E. H. Lee, A. Kim, Y. K. Oh, C. K. Kim, Biomaterials 2005, 26, 205.

[69] M. Cano-Sarabia, A. Angelova, N. Ventosa, S. Lesieur, J. Veciana, J. Colloid Interface Sci. 2010, 350, 10.

[70] L. Kalantzi, K. Goumas, V. Kalioras, B. Abrahamsson, J. B. Dressman, C. Reppas, Pharm. Res. 2006, 23, 165.

[71] C. J. Patton, S. R. Crouch, Anal. Chem. 1977, 49, 464. 


\section{Supporting Information}

\section{LipoBots: urease-nanomotors based on liposomal vesicles for enzyme protection and on- demand triggered motion}

Ana C. Hortelão, ${ }^{\dagger}$ Sonia García-Jimeno, ${ }^{\dagger}$ Mary Cano-Sarabia, ${ }^{\dagger}$ Tania Patiño, ${ }^{\not} \|^{* *}$ Daniel Maspoch $^{\dagger *}$ and Samuel Sanchez ${ }^{\ddagger *}$

*Institute for Bioengineering of Catalonia (IBEC), The Barcelona Institute of Science and Technology (BIST), Baldiri i Reixac 10-12, 08028 Barcelona Spain

${ }^{\dagger}$ Catalan Institute of Nanoscience and Nanotechnology (ICN2), CSIC and The Barcelona Institute of Science and Technology, Universitat Autonoma de Barcelona, Bellaterra, 08193 Barcelona, Spain

"Chemistry Department, University of Rome, Tor Vergata, Via della Ricerca Scientifica, 00133 Rome, Italy

§ Institució Catalana de Recerca i Estudis Avancats (ICREA), Passeig Lluís Companys 23, 08010 Barcelona, Spain 


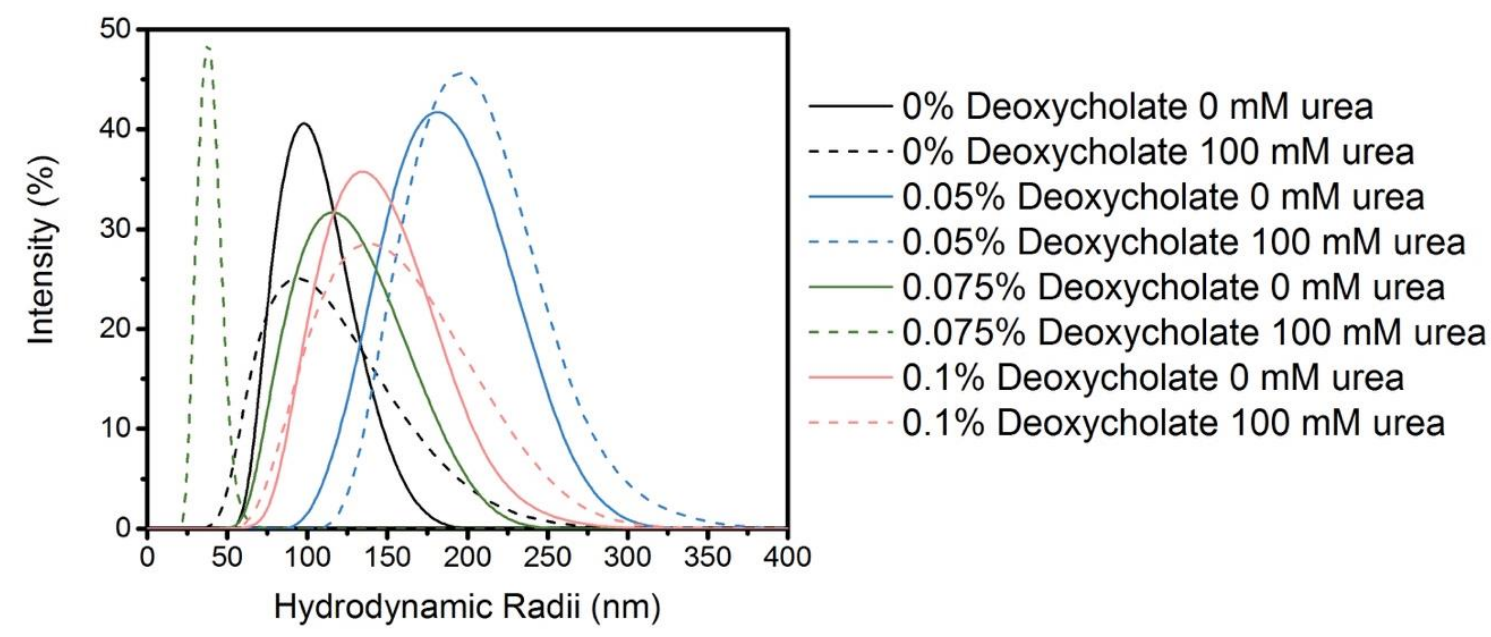

Figure S1. Hydrodynamic size distributions of LB-I in the presence of different concentrations of deoxycholate, in the absence and presence $(100 \mathrm{mM})$ of urea.
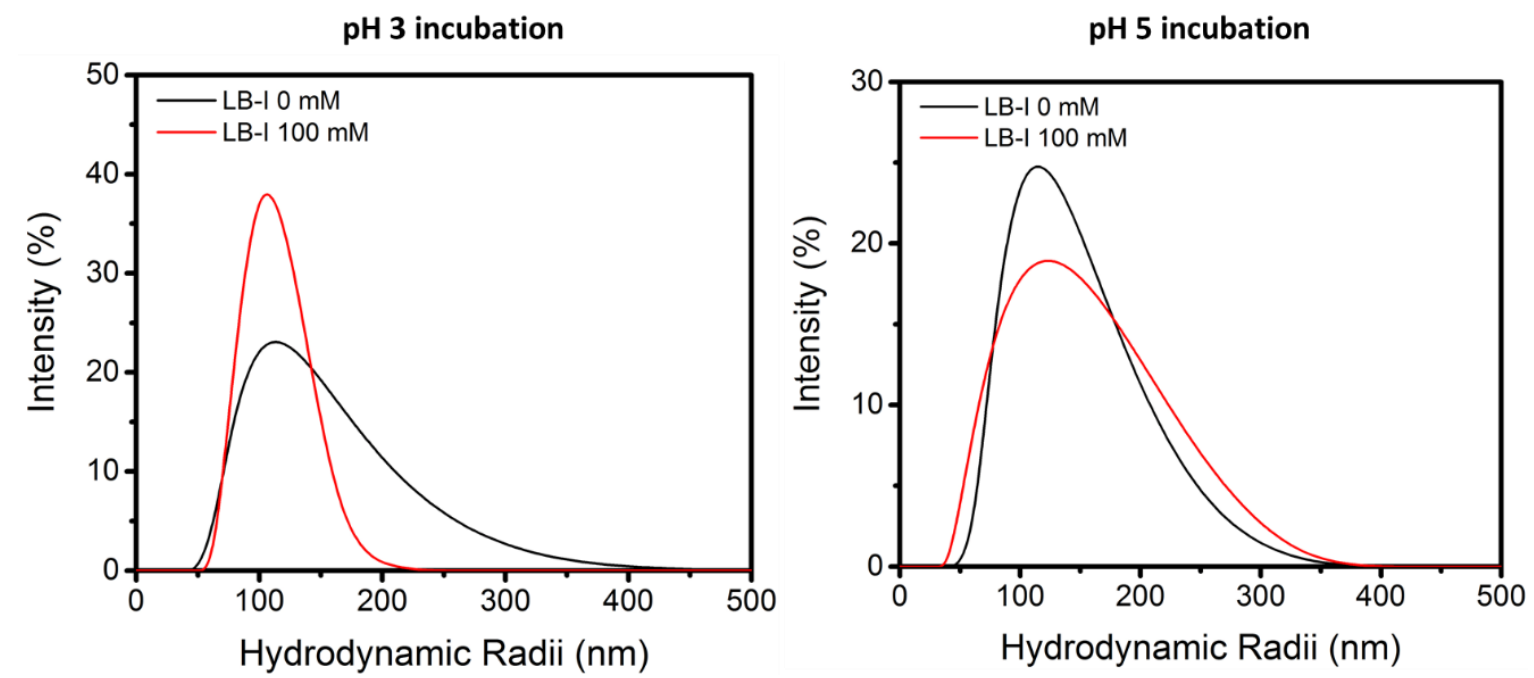

Figure S2. Hydrodynamic size distributions of LB-I after incubation in acidic conditions, $\mathrm{pH} 3$ on the left and $\mathrm{pH} 5$ on the right, in the absence and presence of urea. 

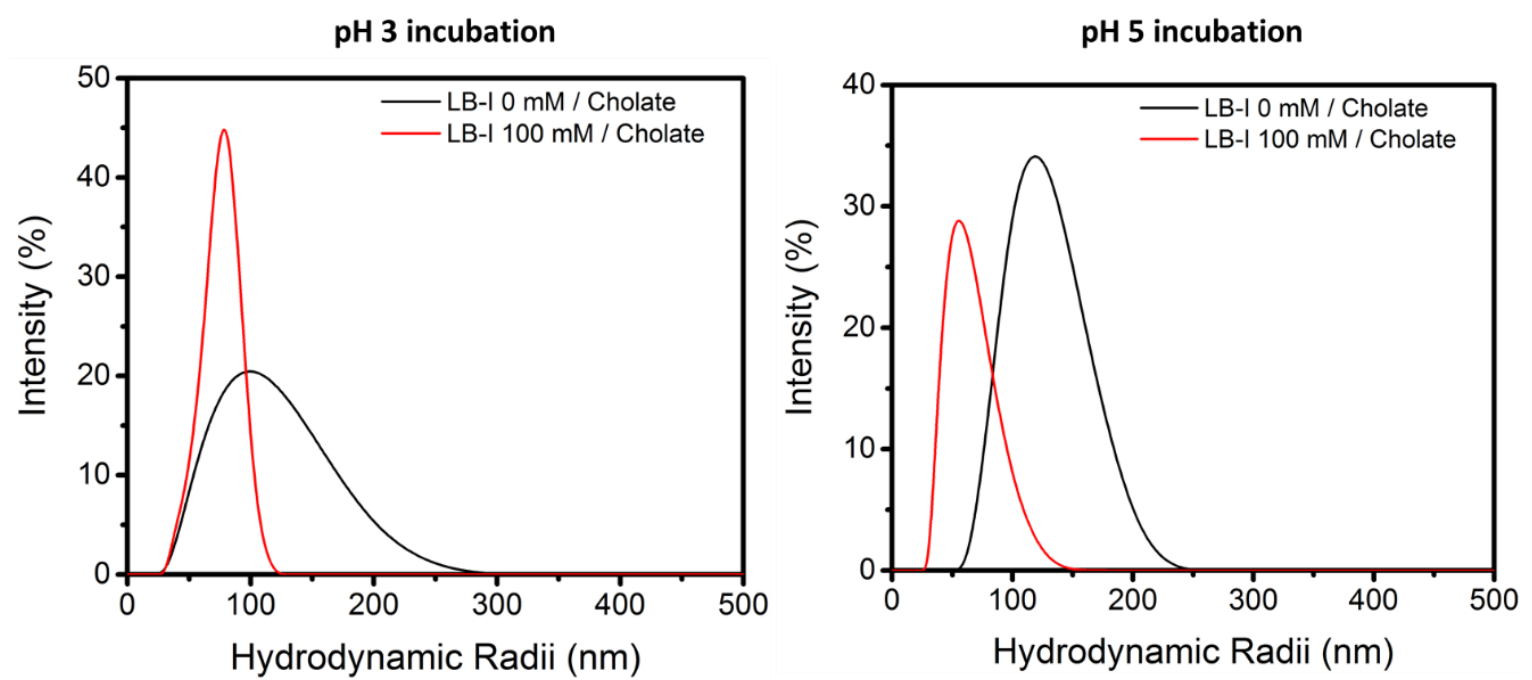

Figure S3. Hydrodynamic size distributions of LB-I after incubation in acidic conditions, $\mathrm{pH} 3$ on the left and $\mathrm{pH} 5$ on the right, in the absence and presence of urea, as well as $0.075 \%(\mathrm{w} / \mathrm{w})$ of sodium deoxycholate.

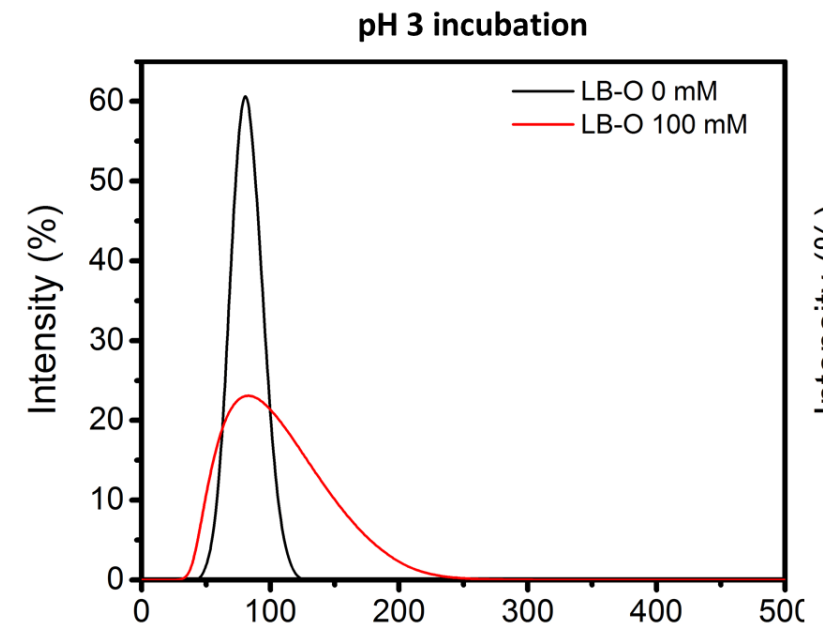

Hydrodynamic Radii $(\mathrm{nm})$

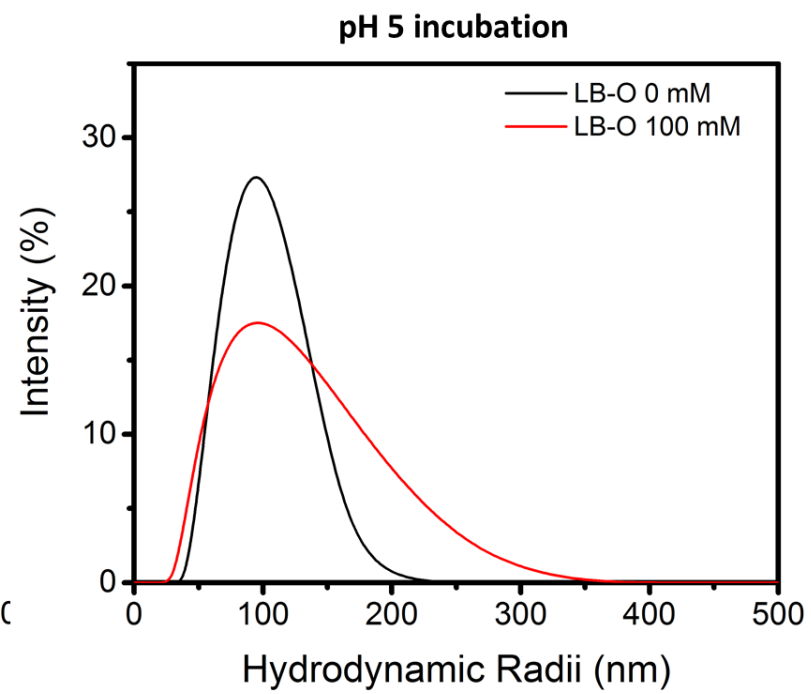

Hydrodynamic Radii (nm)

Figure S4. Hydrodynamic size distributions of LB-O after incubation in acidic conditions, $\mathrm{pH} 3$ on the left and $\mathrm{pH} 5$ on the right, in the absence and presence of urea. 